\title{
REES MATRIX COVERS FOR LOCALLY INVERSE SEMIGROUPS
}

\author{
BY
}

D. B. MCALISTER

\begin{abstract}
A regular semigroup $S$ is locally inverse if each local submonoid $e S e, e$ an idempotent, is an inverse semigroup. It is shown that every locally inverse semigroup is an image of a regular Rees matrix semigroup, over an inverse semigroup, by a homomorphism $\theta$ which is one-to-one on each local submonoid; such a homomorphism is called a local isomorphism. Regular semigroups which are locally isomorphic images of regular Rees matrix semigroups over semilattices are also characterized.
\end{abstract}

Locally inverse semigroups are regular semigroups $S$ in which each local submonoid $e S e, e$ an idempotent, is an inverse semigroup. These semigroups have been extensively studied in recent years; in particular by Nambooripad, Meakin, Pastijn and Byleen. They form a broad class of regular semigroups which includes completely 0-simple semigroups, inverse semigroups and subdirect products of such semigroups as special cases. As well, naturally ordered regular semigroups are locally inverse. An extensive bibliography on locally inverse semigroups will be found in [9].

Pastijn [9] has shown that every locally inverse semigroup divides a semigroup which can be interpreted as an ideal in a Rees matrix semigroup over an $E$-unitary inverse semigroup. More precisely, given a locally inverse semigroup $S$, there is a Rees matrix semigroup $R$, over an $E$-unitary inverse semigroup, and a subsemigroup $T$ of $R$ such that $S$ is a homomorphic image of $T$. Indeed, the semigroup $T$ can be taken as an order ideal of $R$ and the homomorphism $\theta: T \rightarrow S$ is strictly compatible (in the sense of [7]).

On the other hand, Byleen [1] has shown that the 4-spiral semigroup [2] is isomorphic to a Rees matrix semigroup over the bicyclic semigroup. The author [5] has. shown that any locally inverse semigroup $S$, such that $S=S u S$ for some idempotent $u$, is a locally isomorphic image of a regular Rees matrix semigroup over an inverse semigroup. The purpose of this note is to extend the latter result to all locally inverse semigroups.

1. Preliminaries. Let $S$ be a regular semigroup and let $e$ be an idempotent of $S$; then we shall call $e S e$ a local submonoid of $S$. We say that $S$ is locally inverse if each local submonoid of $S$ is an inverse semigroup. If $S$ and $T$ are regular semigroups then we say that a homomorphism $\theta$ of $S$ onto $T$ is a local isomorphism if $\theta$ is one-to-one on each local submonoid of $S$; in this case, we say that $T$ is a locally isomorphic image of $S$.

Received by the editors May 25, 1982.

1980 Mathematics Subject Classification. Primary 20M10.

Key words and phrases. Regular semigroup, inverse semigroup, locally testable semigroup, Rees matrix semigroup, local submonoid, local isomorphism. 
Given any regular semigroup $S$, sets $I$ and $\Lambda$ and a $\Lambda \times I$ matrix $P$ over $S$ we can form the $I \times \Lambda$ Rees matrix semigroup $\Re(S ; I, \Lambda ; P)$ over $S$, with sandwich matrix $P$. Its elements consist of all triples $(i, s, \lambda)$ with multiplication

$$
(i, s, \lambda)(j, t, \mu)=\left(i, s p_{\lambda j} t, \mu\right) .
$$

In general, $\mathfrak{N}(S ; I, \Lambda ; P)$ is not regular. However, the set of all regular elements is a (regular) subsemigroup. We denote this regular semigroup by $\Re \Re(S ; I, \Lambda ; P)$ and call it the regular $I \times \Lambda$ Rees matrix over $S$, with sandwich matrix $P$. It consists of all triples $(i, s, \lambda)$ such that $V(s) \cap p_{\lambda j} S p_{\mu i} \neq \varnothing$ for some $j \in I, \mu \in \Lambda$. The following lemma gives some elementary properties of $\Re \Re(S ; I, \Lambda ; P)$ which we shall find useful in the sequel.

LEMMA 1.1 [5]. Let $S$ be a regular semigroup, $I$ and $\Lambda$ be nonempty sets and let $P$ be a $\Lambda \times I$ matrix over $S$. Then:

(i) $\Re \mathscr{R}(S ; I, \Lambda ; P)=\left\{(i, s, \lambda) \in I \times S \times \Lambda: V(s) \cap p_{\lambda j} S p_{\mu i} \neq \varnothing\right.$ for some $j$ $\in I, \mu \in \Lambda\}$;

(ii) $(i, s, \lambda)$ is idempotent if and only if $s=s p_{\lambda i} s$;

(iii) if $S$ is inverse, then $\Re \Re(S ; I, \Lambda ; P)$ is locally inverse.

Let $S$ be a regular semigroup and let $e, f$ be idempotents in $S$. Then we will denote by $S(e, f)$ the sandwich set of $e$ and $f$. Thus

$$
S(e, f)=\left\{g^{2}=g \in S: e g f=e f, g e=g=f g\right\} .
$$

The sandwich set was introduced by Nambooripad [6] who also introduced the natural partial order $\leqslant$ on a regular semigroup. This is defined as follows: $a \leqslant b$ if and only if $a=a a^{\prime} b$ for some (all) $a^{\prime} \in V(a)$, and $a \in b S$. Although it is not obvious from the definition, $\leqslant$ is left-right symmetric. Indeed, $a \leqslant b$ if and only if $a=e b=b f$ for some idempotents $e, f$ (P. R. Jones, unpublished).

Nambooripad [7] has shown that the following are equivalent for a regular semigroup $S$.

LEMMA 1.2. Let $S$ be a regular semigroup. Then the following are equivalent:

(i) $S$ is locally inverse;

(ii) $|S(e, f)|=1$ for each pair of idempotents $e, f \in S$;

(iii) $\leqslant$ is compatible with multiplication.

A regular semigroup $S$ is called orthodox if the idempotents form a subsemigroup. T. E. Hall [3] has shown that the minimum inverse congruence $\mathcal{O}$ on an orthodox semigroup can be defined as follows:

$$
(a, b) \in \mathcal{Y} \text { if and only if } V(a) \cap V(b) \neq \varnothing ;
$$

in this case $V(a)=V(b)$, where $V(a)$ denotes the set of inverses of $a$ in $S$.

The definition of a local isomorphism $\theta$ of $S$ onto $T$ requires only that $\theta$ is one-to-one on each local submonoid of $S$. However, as the next lemma shows, such a mapping is necessarily one-to-one on each subset of the form $a S b, a, b \in S$.

LEMMA 1.3. Let $\theta$ be a local isomorphism of a regular semigroup $S$ onto a regular semigroup $T$, and let $a, b \in S$. Then $\theta$ is one-to-one on $a S b$. 
Proof. Suppose $x, y \in a S b$ and that $x \theta=y \theta$. Then $x, y$ have inverses $x^{\prime}, y^{\prime}$ in $b^{\prime} S a^{\prime}$ where $a^{\prime} \in V(a), b^{\prime} \in V(b)$. It follows that $\left(x x^{\prime}\right) \theta=\left(y x^{\prime}\right) \theta$ with $x x^{\prime}, y x^{\prime}$ in $a S a^{\prime}$ which is a local submonoid. Hence $x x^{\prime}=y x^{\prime}$ so that $x=y x^{\prime} x$. Similarly, $\left(y^{\prime} x\right) \theta=\left(y^{\prime} y\right) \theta$ gives $y^{\prime} y=y^{\prime} x$ so that $y \in S x$. But then $x=y x^{\prime} x$ implies $x=y$.

Proposition 1.4. Let $S$ be a regular semigroup and let $\mathcal{Y}$ be the minimum inverse congruence on $S$. Then the canonical homomorphism $\mathcal{O}^{\natural}: S \rightarrow S / \mathcal{Y}$ is a local isomorphism if and only if $S$ is locally inverse and orthodox.

Proof. Suppose that $\theta$ is a local isomorphism of $S$ into an inverse semigroup. Let $e, f$ be idempotents in $S$. Then, since idempotents in $T$ commute, $(e f) \theta=($ efef $) \boldsymbol{\theta}$ but $e f$, efef $\in e S f$ so that, since $\theta$ is a local isomorphism, ef $=e f e f$. Thus $S$ is orthodox. Further, since $e S e$ is isomorphic to $(e S e) \theta$, which is an inverse submonoid of $T, e S e$ is inverse for each idempotent $e$. Thus $S$ is locally inverse.

Conversely, suppose that $S$ is locally inverse and orthodox and suppose that $a, b \in e S e$ with $(a, b) \in \mathcal{Y}$. Then $a$ and $b$ have a common inverse $a^{\prime} \in e S e$. However, this means that $a^{\prime}$ has $a, b$ as inverses in $e S e$. Since $e S e$ is inverse, this implies $a=b$. Hence $\mathcal{O}$ is a local isomorphism.

2. The main theorem. In this section, we prove the main theorem of this paper.

THEOREM 2.1. Let $S$ be a regular semigroup. Then $S$ is locally inverse if and only if $S$ is a locally isomorphic image of a regular Rees matrix semigroup over an inverse semigroup.

Let $S$ be a locally inverse semigroup. Fix an idempotent $e \in S$ and, for each idempotent $f \in S$, let $f^{*} \in S(e, f)$; since $S$ is locally inverse, $f^{*}$ is uniquely determined by $f$. For each pair of idempotents $u, v \in S$, let $p_{u, v}$ in $S$ be defined as follows:

$$
p_{u, v}= \begin{cases}u & \text { if } u=v \\ u^{*} v & \text { if } u \neq v\end{cases}
$$

LEMMA 2.2. For idempotents $u, v, f, g$ in $S$, the following are true:

(i) $p_{u, v}=p_{u, v} p_{v, u} p_{u, v}$;

(ii) $p_{u, v} p_{v, g} p_{g, u}$ is idempotent;

(iii) $p_{u, v} p_{v, f} p_{f, g} \leqslant p_{u, g}$ where $\leqslant$ denotes the natural partial order on $S$.

Proof. (i) If $u=v$ then, since $p_{u, u}=u$, the result is clear. Otherwise,

$$
\begin{aligned}
& p_{u, v} p_{v, u} p_{u, v}=u^{*} v v^{*} u u^{*} v=u^{*} v^{*} u^{*} v \text { since } v^{*} \in S(e, v) \text { implies } v^{*}=v v^{*} \text {, } \\
& u^{*} \in S(e, u) \text { implies } u^{*}=u u^{*}, \\
& =u^{*} e \cdot v^{*} e \cdot u^{*} v \quad \text { since } v^{*} \in S(e, v) \text { implies } v^{*}=v^{*} e \text {, } \\
& u^{*} \in S(e, u) \text { implies } u^{*}=u^{*} e, \\
& =u^{*} \cdot e v^{*} \cdot e u^{*} \cdot v \\
& =u^{*} \cdot e u^{*} \cdot e v^{*} \cdot v \\
& \text { since } e u^{*}, e v^{*} \text { are idempotents in } e S e \text { which is inverse, } \\
& =u^{*} e v^{*} v \\
& =u^{*} e v=u^{*} v=p_{u, v} \quad \text { since } v^{*} \in S(e, v) \text { implies } e v=e v^{*} v \text {. }
\end{aligned}
$$


(ii) If $u=v$ then $p_{u, v} p_{v, g} p_{g, u}=p_{u, g} p_{g, u}$ which is idempotent, by (i). Similarly, if $v=g$ or $g=u$, then (i) shows that (ii) is true, so we may suppose $u \neq v, v \neq g$, $g \neq u$. Then

$$
\begin{aligned}
& p_{u, v} p_{v, g} p_{g, u} \cdot p_{u, v} p_{v, g} p_{g, u}=u^{*} v v^{*} g g^{*} u \cdot u^{*} v v^{*} g g^{*} u \\
&=u^{*} v^{*} g^{*} u^{*} v^{*} g^{*} u \text { as in the proof of (i) } \\
&=u^{*} e \cdot v^{*} e \cdot g^{*} e \cdot u^{*} e \cdot v^{*} \cdot g^{*} u \\
&=u^{*} \cdot e v^{*} \cdot e g^{*} \cdot e u^{*} \cdot e v^{*} \cdot g^{*} u \\
&=u^{*} \cdot e u^{*} \cdot e v^{*} \cdot e v^{*} \cdot e g^{*} \cdot g^{*} u \\
& \quad \text { since idempotents in eSe commute } \\
&=u^{*} \cdot e v^{*} \cdot e g^{*} u \\
&=u^{*} v^{*} g^{*} u=p_{u, v} p_{v, g} p_{g, u} .
\end{aligned}
$$

(iii) We consider first the special case of $p_{u, v} p_{v, f} p_{f, g}$ in which one of $u=v, v=f$, $f=g$ holds. In this case, the expression reduces to $p_{u, v} p_{v, g}$.

If $u=v$ or $v=g$ this expression is just $p_{u, g}$ so that $p_{u, v} p_{v, g} \leqslant p_{u, g}$ is certainly true in this case. If $u \neq v, v \neq g$ then

$$
\begin{aligned}
p_{u, v} p_{v, g} & =u^{*} v \cdot v^{*} g=u^{*} v^{*} g=u^{*} \cdot e u^{*} \cdot e v^{*} \cdot g \\
& =u^{*} \cdot e v^{*} \cdot e u^{*} g=u^{*} v^{*} \cdot u^{*} g .
\end{aligned}
$$

If $g=u$ then the expression is just $p_{u, v} p_{v, u}$ which is an idempotent in $u S u$, and so is below $u=p_{u, u}$. Hence the result is valid in this case. Otherwise

$$
p_{u, v} p_{v, g}=u^{*} v^{*} u^{*} g=u^{*} v^{*} u \cdot u^{*} g=u^{*} v^{*} u \cdot p_{u, g}
$$

But $u^{*} v^{*} u=p_{u, v} p_{v, u}$ is an idempotent in $u S u$, by (i), so that, since $u^{*} v^{*} u \leqslant u$ we have $p_{u, v} p_{v, g} \leqslant u p_{u, g}=p_{u, g}$.

Returning to the general case, one sees that if any of $u=v, v=f, f=g$ holds then the inequality reduces to $p_{u, v} p_{v, g} \leqslant p_{u, g}$ which we have just shown to be true. Hence, we may assume $u \neq v, v \neq f, f \neq g$. Then

$$
p_{u, v} p_{v, f} p_{f, g}=u^{*} v v^{*} f f^{*} g=u^{*} v^{*} f^{*} g .
$$

If $u=g$ this is, by (ii), an idempotent in $u S u$ and so is below $u=p_{u, u}$. If $u \neq g$, we can write the expression as

$$
\begin{aligned}
u^{*} e u^{*} v^{*} f^{*} g & =u^{*} \cdot e u^{*} \cdot e v^{*} \cdot e f^{*} \cdot g \quad \text { as in the proof of (i), } \\
& =u^{*} \cdot e v^{*} \cdot e f^{*} \cdot e u^{*} g \quad \text { since idempotents in } e S e \text { commute } \\
& =u^{*} v \cdot v^{*} f \cdot f^{*} u \cdot u^{*} g \\
& =\left[p_{u, v} \cdot p_{v, f} \cdot p_{f, u}\right] p_{u, g} \\
& \leqslant u p_{u, g}=p_{u, g} \text { by (ii) since } p_{u, v} p_{v, f} p_{f, u} \in u S u .
\end{aligned}
$$

Proposition 2.3. Let $W=\{(u, x, v) \in E \times S \times E: x \in u S v\}$ with the multiplication

$$
(u, x, v)(f, y, g)=\left(u, x p_{v, f} y, g\right) .
$$

Then $W$ is an orthodox locally inverse semigroup. 
Proof. First, it is easy to see that $W$ is a regular semigroup; indeed, $(u, x, v)$ has inverse $\left(v, x^{\prime}, u\right)$ where $x^{\prime} \in V(x) \cap v S u$. Suppose that $(u, x, v)$ is idempotent in $W$. Then $x=x p_{v, u} x=x p_{v, u} \cdot p_{u, v} \cdot p_{v, u} x$ where $x p_{v, u}, p_{v, u} x$ are idempotents in $u S u$, $v S v$ respectively. Hence $x \leqslant u \cdot p_{u, v} \cdot v=p_{u, v}$. Conversely, if $x \leqslant p_{u, v}$, then $x=f p_{u, v}$ $=p_{u, v} g$ where $f, g$ are idempotents. Thus

$$
x p_{v, u} x=f p_{u, v} p_{v, u} p_{u, v} g=f p_{u, v} g=x g=x .
$$

Hence $W$ has, for idempotents, the set of triples $\left\{(u, x, v) \in E \times S \times E: x \leqslant p_{u, v}\right\}$.

Suppose that $(u, x, v)$ and $(g, y, h)$ are idempotents in $W$. Then $(u, x, v)(g, y, h)$ $=\left(u, x p_{v, g} y, h\right)$. Now, since $x \leqslant p_{u, v}, y \leqslant p_{g, h}$, we have $x p_{v, g} y \leqslant p_{u, v} p_{v, g} p_{g, h} \leqslant p_{u, h}$ by Lemma 2.2(iii). Hence $(u, x, v)(g, y, h)$ is idempotent. Thus $W$ is orthodox.

To complete the proof, we must show that $W$ is locally inverse; so suppose that $(u, x, v),(u, y, v),(u, z, v)$ are idempotents with $(u, x, v) \leqslant(u, z, v)$ and $(u, y, v)$ $\leqslant(u, z, v)$. Then

$$
\begin{aligned}
(u, x, v)(u, y, v)=\left(u, x p_{v, u} y, v\right) & \\
& =\left(u, x p_{v, u} y p_{v, u} z, v\right) \quad \text { since }(u, y, v) \leqslant(u, z, v) \text { implies } y=y p_{v, u} z, \\
& =\left(u, y p_{v, u} x p_{v, y} z, v\right) \quad \text { since } x p_{v, u}, y p_{v, u} \text { are idempotents in } u S u, \\
& =\left(u, y p_{v, u} x, v\right) \quad \text { since }(u, x, v) \leqslant(u, z, v) \text { implies } x=x p_{v, u} z, \\
& =(u, y, v)(u, x, v) .
\end{aligned}
$$

Thus the idempotents, in each local submonoid of $W$, commute so that $W$ is locally inverse.

Proof of Theorem 2.1. Let $U=W / \mathcal{Y}$ and denote by $\psi$ the natural homomorphism of $W$ onto $U$. Then $U$ is an inverse semigroup and, by Proposition $1.4, \psi$ is a local isomorphism. Now, let $I$ and $\Lambda$ be subsets of $E$ such that $S=\cup\{u S v: u \in I$, $v \in \Lambda\}$ and form the $\Lambda \times I$ matrix $Q$ over $U$ with

$$
q_{v, u}=(v, v u, u) \psi \text { for } v \in \Lambda, u \in I .
$$

We show that $S$ is a locally isomorphic image of $\Re \Re(U ; I, \Lambda ; Q)$. Suppose that $[u,(g, x, k) \psi, v] \in \Re \Re$ then $(g, x, k) \psi \in(u, u w, w) \psi U(z, z v, v) \psi$ for some $w \in$ $\Lambda, z \in I$. Thus $(g, x, k) \psi=(u, u, u) \psi(g, x, k) \psi(v, v, v) \psi$ so that $\Re \Re \subseteq$ $\{[u,(u, x, v) \psi, v]: x \in u S v\}$. On the other hand, if $x \in u S v$ then $x$ has an inverse $x^{\prime} \in g S k$ for some $g \in I, k \in \Lambda$. Now

$$
\begin{aligned}
& {[u,(u, x, v) \psi, v]\left[g,\left(g, x^{\prime}, k\right) \psi, k\right][u,(u, x, v) \psi, v] } \\
&=\left[u,(u, x, v) \psi(v, v g, g) \psi\left(g, x^{\prime}, k\right) \psi(k, k u, u) \psi(u, x, v) \psi, v\right] \\
&=\left[u,\left(u, x \cdot v g \cdot x^{\prime} \cdot k u \cdot x, v\right) \psi, v\right] \\
&=\left[u,\left(u, x x^{\prime} x, v\right) \psi, v\right] \quad \text { since } x \in u S v, x^{\prime} \in g S k, \\
&=[u,(u, x, v) \psi, v] .
\end{aligned}
$$

Hence, $\Re \Re=\{[u,(u, x, v) \psi, v]: x \in u S v\}$.

Since $\psi$ is an isomorphism on each $(u, u, u) W(v, v, v)=\{(u, x, v): x \in u S v\}$, we can define a mapping $\theta$ from $\Re \Re$ onto $S$ by setting $[u,(u, x, v) \psi, v] \theta=x$. Now

$$
\begin{aligned}
{[u,(u, x, v) \psi, v][g,(g, y, k) \psi, k] } & =[u,(u, x, v) \psi(v, v g, g) \psi(g, y, k) \psi, k] \\
& =[u,(u, x v g y, k) \psi, k]=[u,(u, x y, k) \psi, k] .
\end{aligned}
$$


Hence,

$$
\begin{aligned}
{[u,(u, x, v) \psi, v] \theta[g,(g, y, k) \psi, k] \theta } & =x y \\
& =[u,(u, x, v) \psi, v][g,(g, y, k) \psi, k] \theta
\end{aligned}
$$

so that $\theta$ is a homomorphism. Finally, suppose $[u,(u, x, v) \psi, v] \theta=$ $[u,(u, y, v) \psi, v] \theta$. Then, by definition, $x=y$; thus $\theta$ is one-to-one on each local submonoid of $\Re \Re$.

COROLlARY 2.4. Let $S$ be an idempotent generated locally inverse semigroup. Then $S$ is a locally isomorphic image of an idempotent generated regular Rees matrix semigroup over an inverse semigroup.

Let $\mathcal{X}$ be a down directed partially ordered set, with $\mathcal{Y}$ an ideal and subsemilattice of $\mathcal{X}$, and let $G$ be a group which acts on $\mathcal{X}$, on the left, by order automorphisms. Then Pastijn [9] has generalized the construction of $E$-unitary inverse semigroups, given in [4], as follows:

Let $I, \Lambda$ be nonempty sets and let $P$ be a $\Lambda \times I$ matrix over $G$ such that $p_{\lambda i}$ induces an automorphism on $\mathcal{O}$ for each $\lambda \in \Lambda, i \in I$. Then the set

$$
M=\left\{(i, A, g, \lambda) \in I \times \mathcal{O} \times G \times \Lambda: g^{-1} A \in \mathcal{O}\right\}
$$

is a semigroup under the multiplication

$$
(i, A, g, \lambda)(j, B, h, \mu)=\left(i, A \wedge g p_{\lambda_{j}} B, g p_{\lambda_{j}} h, \mu\right) .
$$

Pastijn [9] shows that $M$ is a locally inverse semigroup and that every locally inverse semigroup is a strictly compatible (in the sense of [7]) image of an order ideal and subsemigroup of a semigroup constructed as above.

His construction can be interpreted in terms of regular Rees matrix semigroups. For, with the notation above, let $S=P(G, \mathfrak{X}, \mathcal{Y})$ be the $E$-unitary inverse semigroup constructed from $G, \mathcal{X}, \mathcal{Y}$, as in [4], and let $\tilde{S}=S \cup\{g \in G: g \mathcal{Y}=\mathcal{Y}\}$, where multiplication is extended from $S$ to $\tilde{S}$ by setting $(B, h) \cdot g=(B, h g)$, $g \cdot(B, h)=(g B, g h)$ for $(B, h) \in S, g \in G$. Then $\tilde{S}$ is an inverse semigroup. It is isomorphic to the semigroup obtained by adjoining the invertible elements of the translational hull $\Omega(S)$ of $S$ to $S$. Thus we can form the regular Rees matrix semigroup $R=\Re \Re(\tilde{S} ; I, \Lambda ; P)$ over $\tilde{S}$, using the matrix $P$, above. It is easily seen that the mapping given by $(i, A, g, \lambda) \mapsto(i,(A, g), \lambda)$ is an embedding of $M$ into $R$, indeed onto an ideal of $R$. Hence, Pastijn's result can be rephrased in the following form: Every locally inverse semigroup is a strictly compatible image of a subsemigroup and order ideal of a regular Rees matrix semigroup over an $E$-unitary inverse semigroup.

COROllary 2.5. Let $S$ be a locally inverse semigroup. Then there exist an E-unitary inverse semigroup $T$, a regular Rees matrix semigroup $\Re \Re(T ; I, \Lambda ; P)$ over $T$ and a homomorphism $\phi$ of $\Re \mathscr{R}$ onto $S$ such that $u \phi^{-1}$ is completely simple for each idempotent $u \in S$.

PROof. With the notation introduced in the proof of Theorem 2.1, $S$ is a locally isomorphic image of $\Re \Re(U ; I, \Lambda ; Q)$, under a homomorphism $\theta$. Let $T$ be an 
$E$-unitary inverse semigroup which admits an idempotent separating homomorphism $\zeta$ onto $U$ (such exists, by [4, Theorem 2.4]), and let $p_{\lambda i} \in T$ be such that $p_{\lambda i} \zeta=q_{\lambda i}$ for each $i \in I, \lambda \in \Lambda$. Then the mapping $\tau: \Re \Re(T ; I, \Lambda ; P) \rightarrow \Re \Re(U ; I, \Lambda ; Q)$ defined by $(i, t, \lambda) \tau=(i, t \zeta, \lambda)$ is easily seen to be an onto, idempotent separating homomorphism. Its composite $\phi$ with $\theta$ gives the required homomorphism onto $S$.

In the proof of Theorem 2.1, and Corollary 2.5, we can take $I=E=\Lambda$, where, as usual, $E$ denotes the set of all idempotents of $S$. The next result gives a sufficient condition for a locally inverse semigroup to be isomorphic to a regular Rees matrix semigroup over an inverse semigroup.

Proposition 2.6. Let $S$ be a locally inverse semigroup and suppose that there are sets $I, \Lambda$ of idempotents of $S$ such that:

(i) $S=\bigcup\{u S v: u \in I, v \in \Lambda\}$,

(ii) $u S v \cap g S k \neq \varnothing$ implies $u=g, v=k$, for $u, g \in I, g, k \in \Lambda$.

Then $S$ is isomorphic to an $I \times \Lambda$ regular Rees matrix semigroup over an inverse semigroup.

Proof. Let $U$ be as in the proof of Theorem 2.1. Then the mapping $\theta$ given by $[u,(u, x, v) \psi, v] \theta=x$ is a local isomorphism of $\Re \Re(U ; I, \Lambda ; Q)$ onto $S$. Suppose that $[u,(u, x, v) \psi, v] \theta=[g,(g, y, k) \psi, k] \theta$. Then $x=y \in u S v \cap g S v$. Hence, by (ii), $u=g, v=k$ so that $\theta$ is one-to-one.

As a special case of Proposition 2.6, we have the following useful criterion, which depends only on the biorder structure of the idempotents in a locally inverse semigroup.

THEOREM 2.7. Let $S$ be a locally inverse semigroup in which

(i) each principal left (right) ideal is contained in a maximal principal left (right) ideal;

(ii) distinct maximal principal left (right) ideals are disjoint.

Then $S$ is isomorphic to a regular Rees matrix semigroup over an inverse semigroup.

Proof. Let $I, \Lambda$ be representative sets of idempotent generators for the maximal principal right and left ideals of $S$. Then the hypotheses of Theorem 2.7 imply those of Proposition 2.6. Hence the result follows.

COROLlARY 2.8 (BYLEEN [1]). Let $S$ be a regular semigroup. If the idempotents of $S$ generate a 4-spiral semigroup then $S$ is isomorphic to a $2 \times 2$ Rees matrix semigroup over an inverse semigroup.

3. Locally testable semigroups. A regular semigroup $S$ is said to be locally testable if $e S e$ is a semilattice for each idempotent $e$. Such a semigroup is clearly locally inverse and the construction in $\$ 2$ shows that every locally testable (regular) semigroup is a locally isomorphic image of a regular Rees matrix over a locally testable inverse semigroup. On the other hand, the local structure theorem of [5] shows that if $S$ is a locally testable semigroup, of the form $S=S u S$, then $S$ is a locally isomorphic image of a regular Rees matrix semigroup over a semilattice. 
Pastijn [11] has shown, more generally, that every locally testable semigroup divides a regular Rees matrix semigroup over a semilattice. Thus it is natural to ask if the analog of Theorem 2.1 holds for locally testable semigroups, and semilattices. This, however, is not the case. In this section we give a set of necessary and sufficient conditions to determine when a semigroup is a locally isomorphic image of a regular Rees matrix semigroup over a semilattice.

A subset $Q$ of a regular semigroup $S$ is called a quasi-ideal if $Q S Q \subseteq Q$. It is easy to see that $Q$ is a quasi-ideal of $S$ if and only if it is the intersection of a left ideal and a right ideal of $S$. The following results of Pastijn [11], describe relationships between a locally testable semigroup and its quasi-ideals.

THEOREM 3.1. Let $S$ be a regular semigroup. Then:

(i) $S$ is locally testable if and only if, for each $a \in S, a S a=\{x: x \leqslant a\}$;

(ii) the set $Q(S)$ of quasi-ideals of $S$ is a (regular) locally testable semigroup under subset multiplication;

(iii) if $S$ is locally testable then the mapping $\eta: a \mapsto a S a$ is an isomorphism of $S$ into $Q(S)$; in particular $a S a \cdot b S b=a b S a b$ for all $a, b \in S$.

We shall use these results of Pastijn and the next lemma in the proof of the main result of this section.

LEMMA 3.2. Let $S$ be a locally testable semigroup and suppose that $S e S \cap S f S=S g S$ for some idempotents $e, f, g$ in $S$. Then $e S f=z S z$ for some $z \in S$.

Proof. Since $g \in S e S$ there exist $x \in S, x^{\prime} \in V(x)$ such that $x^{\prime} x=g, x x^{\prime} \leqslant e$; also $y \in S, y^{\prime} \in V(y)$ such that $y y^{\prime}=g, y^{\prime} y \leqslant f$. Then $x \Re x y \& y$ so that $S x y S=S g S$, and $x y \in e S f$ since $e x=e x x^{\prime} x=x x^{\prime} x=x, y=y f$. Put $z=x y$, we shall show that $e S f=z S z$.

Let $a \in e S f$ then $a \in S g S=S u S$ where $u^{2}=u \Re x y$. Then there exists $a^{\prime} \in V(a)$ such that $a a^{\prime} \in e S e$. Thus $a a^{\prime} \in S u S$ implies $a a^{\prime}=e a a^{\prime} e \in e S e \cdot u \cdot e S e$ so that, since $e S e$ is a semilattice, $a a^{\prime} \leqslant e u e=u e$. It follows that uea $=a$ and thus $a \in u S=$ $x y S$. Dually, $a \in S x y$ so that $a=a a^{\prime} a \in x y S x y$. Hence $e S f \subseteq z S z$ but, clearly, we have $z S z \subseteq e S f$ so equality prevails.

THEOREM 3.3. Let $S$ be a regular semigroup. Then the following statements are equivalent:

(i) $S$ can be embedded as a quasi-ideal in a locally testable semigroup $T$ such that $T=T u T$ for some $u \in T$;

(ii) $S$ is locally inverse and eSf has a greatest member for each $e, f \in S$;

(iii) $S$ is locally testable and the principal ideals form a semilattice under inclusion;

(iv) $S$ is a locally isomorphic image of a regular Rees matrix semigroup over a semilattice.

Proof. We shall show first that (iv) $\Rightarrow$ (ii) $\Rightarrow$ (i) $\Rightarrow$ (iv).

Let $R=\Re \Re(U ; I, \Lambda ; P)$ be a regular Rees matrix semigroup over a semilattice and let $(i, x, \lambda),(f, y, \mu)$ be idempotents in $R$; thus $x=x p_{\lambda i} x$ and $y=y p_{\mu j} y$. Then 
$(i, z, \mu) \in(i, x, \lambda) R(j, u, \mu)$ if and only if $z=x p_{\lambda i} z=z p_{\mu j} y$. Since $U$ is a semilattice, this occurs if and only if $z \leqslant x p_{\lambda i} p_{\mu j} y$. Hence

$$
(i, x, \lambda) R(j, y, \mu)=\left\{(i, z, \mu): z \leqslant x p_{\lambda i} p_{\mu_{j}} y\right\}
$$

has a greatest element.

Suppose now that $\theta$ is a homomorphism of $R$ onto a regular semigroup $s$ and let $e$, $f$ be idempotents in $S$. Then, by Lallement's lemma, there are idempotents $u, v \in R$ such that $u \theta=e, v \theta=f$; consequently $(u R v) \theta=e S f$. Let $x \in e S f$, then there exists $y \in u R v$ with $y \theta=x$. Now, $y \leqslant m$, where $m$ is the greatest element of $u R v$, so that, since homomorphisms preserve the natural partial order, $x=y \theta \leqslant m \theta \in e S f$. Hence $e S f$ has a greatest element. Further, since $R$ is a regular Rees matrix semigroup over an inverse semigroup, $R$ is locally inverse. Therefore, so is $S$ and so (iv) implies (ii).

Suppose (ii) holds. Then, firstly, $S$ is locally testable. For if $m$ denotes the maximum member of $e S e$ then, from the definition of $\leqslant$, we find that $e=m$. Thus $x \in e S e$ implies $x \leqslant e$ which, in turn implies $x=u e=e v$ for some idempotents $u, v$. This gives $x^{2}=u e x=u x=u \cdot u e=u e=x$ so that $e S e$ is a band. Since $S$ is locally inverse, it follows that $S$ is locally testable. Hence, by Theorem $3.1, S$ can be embedded in the semigroup $Q(S)$ of quasi-ideals of $S$.

Let $\mathcal{T} \subseteq Q(S)$ consist of all quasi-ideals of $S$ of the forms

$$
S, a S, S a, S a S, a S a \text { for } a \in S \text {. }
$$

It follows from (ii) that $a S b=c S c$ for some $c \in S$, for any $a, b \in S$. Thus, it is easy to see that $\mathcal{T}$ is a regular subsemigroup of $Q(S)$ into which $S$ can be embedded as a quasi-ideal. Further, $\mathcal{T}=\mathcal{T} S \mathcal{T}$, where $S$ is an idempotent of $\mathcal{T}$.

(i) $\Rightarrow$ (iv) For each idempotent $e$ in $S$ there exist $r_{e}, r_{e}^{\prime} \in V\left(r_{e}\right)$ in $T$ such that $r_{e} r_{e}^{\prime}=e, r_{e}^{\prime} r_{e} \omega u$. Let $p_{f, e}=r_{f} r_{e}^{\prime} \in f T e \subseteq S$. Then, for $x \in e S f$, we have

$$
x p_{f, e} x=r_{e} \cdot r_{e}^{\prime} x r_{f} \cdot r_{e}^{\prime} x r_{f} \cdot r_{f}^{\prime}=r_{e} \cdot r_{e}^{\prime} x r_{f} \cdot r_{f}^{\prime}=x
$$

since $r_{e}^{\prime} x r_{f} \in u T u$ which is a semilattice.

Let

$$
W=\{(e, x, f) \in E \times S \times E: x \in e S f\}
$$

under the multiplication $(e, x, f)(g, y, h)=\left(e, x p_{f g} y, h\right)$. Then, since $x p_{f, e} x=x$ for $x \in e S f, W$ is a band and, as in the proof of Theorem 2.1, it is locally inverse. Hence the canonical homomorphism $\mathcal{Y}^{\natural}: W \rightarrow W / \mathcal{Y}$ is a local isomorphism onto a semilattice. It follows, as in the proof of Theorem 2.1 , that $S$ is a locally isomorphic image of a regular Rees matrix semigroup over $W / \mathcal{Y}$.

Finally, we show the equivalence of (iii) with the other three statements. Suppose (i) and thus (ii) and (iv) hold. Then $S \mathscr{T} S=\left\{S e S: e^{2}=e\right\} \cup\{S\}$ so that this is a semilattice under subset multiplication. Indeed, $S e S \cdot S f S=S p_{e, f} S$ where $p_{e, f}=$ $\max \{x: x \in e S f\}$. Since $\operatorname{SeS} \leqslant S f S$ if and only if $\operatorname{SeS}=S e S \cdot S f S$ it is easy to see that $\leqslant$ coincides with inclusion. Hence (iii) holds.

Conversely, suppose that (iii) holds. Then, by Lemma 3.2, given idempotents, $e, f, e S f=z S z$ for some $z \in S$ and so, by Theorem 3.1,eSf $=\{x \in S: x \leqslant z\}$ has a greatest element. Thus (ii) holds. 
COROllary 3.4. Under the conditions of Theorem 3.3, $S$ is a locally isomorphic image of a regular Rees matrix semigroup over the semilattice of principal ideals.

Proof. From the form of $\mathcal{Y}$ on a locally inverse band, it follows that, if we take $\mathcal{T}$ for $T$ in the proof of Theorem 3.3, then $W / \mathcal{Y}$ is isomorphic to the semilattice of principal ideals of $S$.

Example 3.5, which follows, shows that the principal ideals of a locally testable semigroup need not form a semilattice under inclusion. Hence the semigroup need not be a locally isomorphic image of a regular Rees matrix semigroup over a semilattice.

EXAMPLE 3.5. Let $S=\{1,2\} \times[0,1) \times\{1,2\} \cup\{(i, 1, i): i=1,2\}$ under the multiplication

$$
(i, x, j)(u, y, v)= \begin{cases}(i, x \wedge y, v) & \text { if } j=u, \\ (i, 0, v) & \text { otherwise. }\end{cases}
$$

Then $S$ is a locally testable semigroup; the idempotents are the triples $(i, x, i)$, $i=1,2, x \in[0,1]$. Since $(1,1,1) S(2,1,2)=\{(1, x, 2): x \in[0,1)\}$, we see that $(1,1,1) S(2,1,2)$ does not have a greatest element so that (ii) of Theorem 3.3 does not hold.

The distinct principal ideals of $S$ are generated by the idempotents $(1, x, 1), x \leqslant 1$ and $(2,1,2)$. We have

$$
\begin{aligned}
& (1, x, 1) \leqslant_{g}(1, y, 1) \text { if and only if } x \leqslant y, \\
& (1, x, 1) \leqslant_{g}(2,1,2) \text { if and only if } x<1 .
\end{aligned}
$$

Hence $S(1,1,1) S$ and $S(2,1,2) S$ have no greatest lower bound.

The situation in Example 3.5 can occur only because the partially ordered set of principal ideals is not updirected. Indeed, we have

Proposition 3.6. Let $S$ be a locally testable semigroup in which the set of principal ideals is updirected. Then $S$ is a locally isomorphic image of a regular Rees matrix semigroup over a semilattice.

Proof. Let $e, f$ be idempotents of $S$. Then there is an idempotent $u$ in $S$ such that $\{e, f\} \subseteq S u S$. Thus there exist $r_{e}, r_{f} \in S, r_{e}^{\prime} \in V(e), r_{f}^{\prime} \in V(f)$ such that $r_{e} r_{e}^{\prime}=e$, $r_{e}^{\prime} r_{e} \omega u, r_{f} r_{f}^{\prime}=f, r_{f}^{\prime} r_{f} \omega u$. Let $x \in e S f$, then $x=r_{e} r_{e}^{\prime} x r_{f} r_{f}^{\prime}$ where $r_{e}^{\prime} x r_{f} \in u S u$ which is a semilattice; so that $r_{e}^{\prime} x r_{f} \leqslant u$. Hence $x \leqslant r_{e} u r_{f}^{\prime}=r_{e} r_{f}^{\prime}$ so that $e S f$ has greatest element $r_{e} r_{f}^{\prime}$. The result now follows from Theorem 3.3.

COROLlaRY 3.7. Let $S$ be a regular semigroup. Then $S$ is a locally isomorphic image of a regular Rees matrix semigroup over a chain if and only if it is locally testable and its principal ideals form a chain under inclusion.

4. Acknowledgements and closing remarks. A weaker version of Theorem 2.1 was previously obtained by T. E. Hall and the author (unpublished). This result was obtained by embedding a locally inverse semigroup $S$ as a subsemigroup and order ideal in a locally inverse semigroup $T$, of the form $T=T u T$ for some idempotent $u$. The local structure theorem of [5] was then used to express $S$ as a locally isomorphic 
image of a subsemigroup and order ideal of a regular Rees matrix semigroup over the inverse semigroup $u T u$.

The existence of an embedding of $S$ into $T$, as above, depends strongly on Hall's result, announced at the Nebraska Semigroup Conference, September, 1980, which showed that any regular semigroup $U$ can be embedded in a regular semigroup $V$ so that idempotents which are potentially $\mathscr{D}$-related in $U$ are actually $\mathscr{D}$-related in $V$. Hall and the author (also unpublished) had previously shown that any locally inverse semigroup $S$, with zero, could be obtained as a locally isomorphic image of a regular Rees matrix semigroup over an inverse semigroup. Their proof of this result also depends on embedding $S$ in a locally inverse semigroup $T$ of the form $T=T u T$. The same argument extends to give the following result which was also found by Margolis (letter to the author) using different methods.

THEOREM 4.1. Let $\mathcal{C}$ be a class of regular semigroups with zero and suppose $C$ has the following properties:

(i) if $C \in \mathcal{C}$ then $C^{1} \in \mathcal{C}$;

(ii) the 0-direct union of members of $\mathcal{C}$ is also in $\mathrm{C}$;

(iii) $C \in \mathcal{C}$ if and only if $I G(C)$, the idempotent generated subsemigroup of $C$, is also in $\mathrm{C}$.

Let $S$ be a regular semigroup which is locally in $C$. Then $S$ is a locally isomorphic image of a regular Rees matrix semigroup over a member of $C$.

I am grateful to both Hall and Margolis for interesting discussions on the problem considered in this paper.

Finally, one can obtain any locally inverse semigroup as a divisor of a regular Rees matrix semigroup over an inverse semigroup in the following natural fashion:

Let $S$ be a locally inverse semigroup and denote by $\hat{S}$ the inverse semigroup of all one-to-one partial right translations of $S$. For each pair of idempotents $e, f \in S$ and $g \in S(e, f)$ the mapping $p_{e, f}: S e g \rightarrow S g f$ defined by $x p_{e, f}=x g f$ belongs to $\hat{S}$; this does not depend on $S$ being locally inverse. Thus, we can form the regular Rees matrix semigroup $R=\Re \Re(\hat{S} ; E, E, P)$ over $\hat{S}$; here $E$ is the set of idempotents of $S$ and $p_{e, f}$ is the $e, f$ entry of $P$.

When $S$ is locally inverse, $R^{p}=\{(e, \alpha, f) \in R: \Delta \alpha$ is principal $\}$ is a regular subsemigroup of $R$ and it can be shown that $S$ is a locally isomorphic image of $R^{p}$. If further, the intersection of principal left ideals of $S$ is either empty or principal then $R^{p}$ is, in fact, a regular Rees matrix semigroup over an inverse semigroup. This gives an alternative proof to Theorem 2.1 , for such a semigroup. The class of these semigroups includes E-unipotent regular semigroups, which have been considered by several authors.

\section{REFERENCES}

1. K. Byleen, Regular four-spiral semigroups, idempotent-generated semigroups and the Rees construction, Semigroup Forum 22 (1981), 97-100.

2. K. Byleen, J. Meakin and F. Pastijn, The fundamental four sprial semigroup, J. Algebra 54 (1978), 6-26. 
3. T. E. Hall, On regular semigroups whose idempotents form a subsemigroup, Bull. Austral. Math. Soc. 1 (1969), 195-208.

4. D. B. McAlister, Groups, semilattices and inverse semigroups, Trans. Amer. Math. Soc. 192 (1974), $227-244$.

5. __ Regular Rees matrix semigroups and regular Dubreil-Jacotin semigroups, J. Austral. Math. Soc. Ser. A 31 (1981).

6. K. S. S. Nambooripad, The structure of regular semigroups. I, Mem. Amer. Math. Soc. No. 224 (1979).

7. , The natural partial order on a regular semigroup, Proc. Edinburgh Math. Soc. (2) 23 (1980), 249-260.

8. F. Pastijn, Rectangular bands of inverse semigroups, Simon Stevin 56 (1982), 1-97.

9. ___ The structure of pseudo-inverse semigroups, Trans. Amer. Math. Soc. 273 (1982), 631-655.

10. __ Structure theorems for pseudo-inverse semigroups (Proc. Sympos. Regular Semigroups, N.I.U., April, 1979), Dept. of Math. Sci., Northern Illinois University.

11. __ Regular locally testable semigroups as semigroups of quasi ideals, Acta Math. Acad. Sci. Hungar. 36 (1980), 161-166.

Department of Mathematical Sciences, NoRThern Illinois University, DeKalb, Illinois 60115 